\title{
Association of Lipid Parameters with the Risk of Chronic Kidney Disease: A Longitudinal Study Based on Populations in Southern China
}

This article was published in the following Dove Press journal: Diabetes, Metabolic Syndrome and Obesity: Targets and Therapy

\author{
Xin Wang* \\ Haishan Chen* \\ Xiaofei Shao \\ Chongxiang Xiong \\ Guobao Hong \\ Jianhui Chen \\ Xiaolin Li \\ $\mathrm{Xu}$ You \\ Peichun Gao \\ Yunying Chen \\ Ziliang Zou \\ Jing Ning \\ Hua Xiao \\ Hequn Zou \\ Lixin Wei
}

Department of Nephrology, The Third Affiliated Hospital, Southern Medical University, Guangzhou 510630, People's Republic of China

*These authors contributed equally to this work
Objective: To investigate which plasma lipid parameters are useful for detecting chronic kidney disease (CKD) in a Chinese population without known CKD or renal impairment.

Methods: This was a prospective study. In southern Chinese cities from 2012 to 2013, a total of 1037 subjects aged $\geq 18$ years old received a survey. Logistic regression and multiple linear regression analyses were performed. The lipid parameters studied included total cholesterol (TC), triglycerides (TG), high-density lipoprotein cholesterol (HDL-C), non-high-density lipoprotein cholesterol (nHDL-C), TG/HDL-C ratio, TC/HDL-C ratio and nHDL-C/HDL-C ratio. Results: After adjusting for confounding factors, the fourth percentile of logTG/HDL-C was observed to be an independent risk factor for CKD ( $\mathrm{OR}=2.453, \mathrm{P}<0.001)$, and the highest quantile of the logTG/HDL-C ratio was associated with a higher prevalence of $\mathrm{CKD}(\mathrm{P}<0.05)$. This risk was reduced when the model was adjusted with Insulin resistance (IR) $(\mathrm{OR}=2.034, \mathrm{P}<$ $0.05)$. In the group of women, glucose metabolism disorders, high uric acid, and obesity, this risk was increased. Multiple regression models showed that log TG and nonHDL-C/HDL-C were negatively correlated with eGFR $(\mathrm{P}<0.05)$, while $\log$ TG and TC were positively correlated with $\log \mathrm{ACR}(\mathrm{P}<0.05)$. The area under the curve $(\mathrm{ROC})$ of $\lg \mathrm{TG} / \mathrm{HDL}$ was $0.623(\mathrm{p}<0.001)$.

Conclusion: The serum $\log \mathrm{TG} / \mathrm{HDL}-\mathrm{C}$ ratio is the only suitable predictor of $\mathrm{CKD}$, and IR may be the mechanism. This risk needs to be controlled in a specific population. Log TG and nonHDL-C/HDL-C were negatively correlated with eGFR, while log TG and TC were positively correlated with $\log \mathrm{ACR}$.

Keywords: lipid, chronic kidney disease, CKD, urinary albumin to creatinine ratio, ACR, estimated glomerular filtration rate, eGFR

\section{Introduction}

The prevalence and mortality of CKD have risen worldwide, and it has become a global public health issue. ${ }^{1}$ It is reported that the prevalence of ESRD in the United States will increase by $18 \%$ by $2030 .^{2}$ End-stage renal disease (ESRD) patients require long-term hemodialysis treatment, which brings significant economic burden to society. Therefore, it is necessary to identify and control the risk factors to prevent CKD. It is well known that lipids can cause atherosclerosis and increase the risk of cardiovascular disease. Cardiovascular disease and CKD often share common risk factors, so lipids may also be a risk factor for CKD.,

However, there are few data on the risk of lipid-related CKD in the Chinese population. The aim of this study was to prospectively explore the association of serum lipid parameters with CKD in healthy Chinese populations. 


\section{Materials and Methods}

\section{Study Population}

We conducted this cohort study between June 2012 and April 2018. After excluding patients with CKD, other primary nephropathy, and important comorbidities that may affect survival, as well as those taking lipid-lowering drugs at baseline, a total of 1037 volunteers (358 males and 679 females) participated in the study in six communities in Wanzhai Town, Zhuhai City, South China.

\section{Ethics Statement}

The study was conducted in accordance with the Declaration of Helsinki and was approved by the Ethics Committee of the Third Affiliated Hospital of Southern Medical University. All subjects signed a written informed consent form.

\section{Data Collection}

All investigators who participated in this study were specially trained. The sociodemographic data of volunteers were collected using a questionnaire survey, including data on age, gender, smoking, alcohol consumption, current medication, personal history and family history.

Waist circumference was measured mid-way between the lower rib edge of the mid-line and top of the hip bone. Blood pressure was measured using a mercury sphygmomanometer to measure the blood pressure of the left arm of the volunteer 3 times, and the average value was taken.

\section{Laboratory Inspection}

After 12 hrs of fasting, blood samples were collected by venipuncture, and urine samples were collected at the same time. All samples were shipped to the testing center of the Third Affiliated Hospital of Southern Medical University and analyzed within $3 \mathrm{hrs}$.

Insulin was determined by electrochemiluminescence (Roche cobas e601, Roche). Fasting blood glucose was measured using standard enzymatic methods. CRP (Roche cobas 6000 , Orion) was measured by the immunoturbidimetric assay. The blood lipid test method was the colorimetric method (Roche cobas 6000). Serum creatinine was assessed using the modified Jaffe's method (Roche cobas 6000 , Roche). SUA levels were measured by colorimetry (Roche Diagnostics, Mannheim, Germany). The ACR value was calculated by measuring the albumin and creatinine concentrations of random urine samples.

\section{Definition}

Hypertension is defined as systolic blood pressure $\geq 140$ $\mathrm{mmHg}$, diastolic blood pressure $\geq 90 \mathrm{mmHg}$, a history of hypertension and/or the use of antihypertensive drugs. A disorder of glucose metabolism is defined as a fasting blood glucose $\geq 6.1 \mathrm{mmol} / \mathrm{L}$, a history of diabetes and/or the use of a hypoglycemic agent. Obesity is defined as a waist circumference $\geq 90 \mathrm{~cm}$ in males or $\geq 80 \mathrm{~cm}$ in females. Hyperuricemia is defined as a serum uric acid $\geq 420 \mu \mathrm{mol} / \mathrm{L}$ in males or $\geq 360 \mu \mathrm{mol} / \mathrm{L}$ in females. The HOMA-IR calculation formula was as follows: fasting blood glucose level $(\mathrm{FPG}, \mathrm{mmol} / \mathrm{L}) \times$ fasting insulin level $(\mathrm{FINS}, \mathrm{mU} / \mathrm{L}) / 22.5$.

The estimated glomerular filtration rate (eGFR) was determined using the modified MDRD equation: $175 \times$ (Scr) $1.234 \mathrm{x}$ (age) - $0.179 \mathrm{x}$ (if female, $\mathrm{x} 0.79$ ). CKD is defined as $\mathrm{ACR} \geq 3(\mathrm{mg} / \mathrm{mmol})$ and/or eGFR $<60\left(\mathrm{~mL} / \mathrm{min} / 1.73 \mathrm{~m}^{2}\right)$ measured at least 90 days apart (where ACR or eGFR within a single normal value is recorded as 0 ).

In this study, the lipid parameters we studied were triglycerides (TG), total cholesterol (TC), high-density lipoprotein (HDL), non-high-density lipoprotein (nHDL), ratio of triglycerides to high-density lipoprotein (TG/HDL), ratio of TC to HDL (TC/HDL), high-density lipoprotein and non-highdensity lipoprotein ratio (nHDL/HDL). Non-HDL-c is calculated as TC minus HDL-c. TC/HDL-C is calculated as TC divided by HDL-C. The other two lipid ratios were also determined using the same calculation method.

\section{Data Analysis}

All data were analyzed using SPSS (version 20) and MedCalc (v19.0.7). Bilateral p values $<0.05$ were considered statistically significant.

Continuous variables with normal distributions are expressed as the means \pm standard deviations; otherwise, they are represented as intermediate and quartile ranges. The categorical variables are expressed as percentiles.

The variance test was used to compare the mean differences between groups, while the Kruskal-Wallis $H$-test was used to compare the median between groups. The Pearson chisquare $\left(\chi^{2}\right)$ test was used to compare categorical variables.

To amplify the effect of lipid parameters on the OR value of CKD risk, we performed a quartile-based categorical variable transformation on lipid parameters (mentioned above) in the logistic regression analysis. To rule out the collinear effects between the independent variables, we used binomial regression analysis of the LR method to analyze the independent risk factors for CKD. The linear regression 
analysis method of the forward method was used to analyze the relationship between baseline lipid parameters and ACR and eGFR. Therefore, only statistically significant lipid parameter variables are shown in the results. Variables with a skewed distribution, including TG, TG/HDL-C and $\mathrm{ACR}$, were log-transformed before being analyzed.

\section{Results}

Table 1 shows the baseline characteristics of the cohort. A total of 1037 participants (mean age $53 \pm 12$ years) were included in the analysis, only $34.5 \%$ of whom were male.
The baseline characteristics of the cohort were significantly different $(\mathrm{P}<0.05)$ except for smoking, drinking, waist circumference (WC), education and physical activity. Patients with a high triglyceride level were older than patients with a low triglyceride level. As the triglyceride stratification increased, participants had higher uric acid and blood glucose levels, higher SBP and DBP, and higher CRP, while eGFR was decreased. Similarly, in addition to HDL, other lipid parameters also increased with increasing TG.

Logistic regression analysis was used to clarify the longitudinal relationship between the quartile of lipid

Table I The Clinical and Demographic Characteristics of the Study Population Stratified by the Quartile of Baseline Triglycerides

\begin{tabular}{|c|c|c|c|c|c|c|}
\hline \multirow[t]{2}{*}{ Parameter Values } & \multirow[t]{2}{*}{ All $(n=1149)$} & \multicolumn{4}{|c|}{ Quartiles of Total Triglycerides at Baseline [mmol/L] } & \\
\hline & & $\begin{array}{l}\leq 4.71 \\
(n=269)\end{array}$ & $\begin{array}{l}>4.7 I \text { to } \leq 5.37 \\
(n=263)\end{array}$ & $\begin{array}{l}>5.37 \text { to } \leq 6.10 \\
(n=253)\end{array}$ & $\begin{array}{l}>6.10 \\
(n=252)\end{array}$ & \\
\hline Age [years] & $52.63 \pm 12.17$ & $48.01 \pm 13.85$ & $51.98 \pm 11.37$ & $53.98 \pm 11.39$ & $56.89 \pm 9.88$ & $<0.001$ \\
\hline Male, $\mathrm{n}[\%]$ & $35834.50)$ & $103(38.30)$ & $82(31.20)$ & $100(39.50)$ & $73(29.00)$ & 0.026 \\
\hline Smoking [\%] & $38(3.70)$ & $14(5.20)$ & II (4.20) & $7(2.80)$ & $6(2.40)$ & 0.288 \\
\hline Drinking [\%] & $47(4.50)$ & $10(3.70)$ & $9(3.40)$ & $14(5.50)$ & $14(5.60)$ & 0.501 \\
\hline Education attainment High school or above [\%] & $379(36.55)$ & $116(42.65)$ & $85(32.08)$ & $90(35.02)$ & $88(36.21)$ & 0.075 \\
\hline Physical activity [\%] & $466(44.94)$ & $129(47.43)$ & $120(45.28)$ & $113(43.97)$ & $104(42.80)$ & 0.743 \\
\hline Waist circumference [cm] & $84.77 \pm 36.19$ & $83.42 \pm 46.55$ & $85.99 \pm 52.67$ & $84.64 \pm 9.26$ & $85.03 \pm 10.21$ & 0.886 \\
\hline Systolic blood pressure (SBP) [mmHg] & $\mid 27.01 \pm 18.14$ & $122.68 \pm 20.14$ & $127.06 \pm 16.73$ & $127.72 \pm 18.6$ & $130.96 \pm 18.68$ & $<0.001$ \\
\hline Diastolic blood pressure (DBP) $[\mathrm{mmHg}]$ & $77.52 \pm 11.18$ & $75.06 \pm 11.99$ & $78.00 \pm 10.16$ & $78.24 \pm 12.07$ & $78.91 \pm 9.93$ & $<0.001$ \\
\hline Fasting plasma glucose [mmol/L] & $4.96 \pm 0.98$ & $4.79 \pm 0.77$ & $4.86 \pm 0.71$ & $5.00 \pm 1.00$ & $5.19 \pm 1.31$ & $<0.001$ \\
\hline Serum uric acid [umol/L] & $345.33 \pm 91.29$ & $333.02 \pm 92.43$ & $336.49 \pm 91.81$ & $351.54 \pm 88.92$ & $361.45 \pm 89.40$ & $<0.001$ \\
\hline $\begin{array}{l}\text { Estimated glomerular filtration rate (eGFR) } \\
{\left[\mathrm{mL} / \mathrm{min} / 1.73 \mathrm{~m}^{2}\right]}\end{array}$ & $100.64 \pm 20.55$ & $104.09 \pm 22.43$ & $102.66 \pm 22.26$ & $98.47 \pm 18.98$ & $96.99 \pm 17.04$ & $<0.001$ \\
\hline $\begin{array}{l}\text { Urinary microalbumin to creatinine ratio (ACR) } \\
\text { [mg/mmol] }\end{array}$ & $0.91(0.65-1.34)$ & $0.94(0.64-1.34)$ & $0.85(0.63-1.28)$ & $0.87(0.62-1.23)$ & $0.98(0.74-1.43)$ & 0.004 \\
\hline C-reactive protein [mg/L] & $0.92(0.45-2.08)$ & $0.71(0.37-1.65)$ & $0.80(0.39-1.81)$ & $\mathrm{I} .02(0.50-2.5 \mathrm{I})$ & $1.13(0.53-2.49)$ & $<0.001$ \\
\hline HOMA-IR & $1.78(1.20-2.71)$ & $1.58(1.06-2.5 I)$ & $1.66(1.20-2.68)$ & $1.83(1.26-2.90)$ & $2.01(1.23-2.80)$ & $<0.001$ \\
\hline Total cholesterol (TC) $[\mathrm{mmol} / \mathrm{L}]$ & $5.47 \pm 1.04$ & $5.00 \pm 0.89$ & $5.44 \pm 0.97$ & $5.60 \pm 1.01$ & $5.86 \pm 1.12$ & $<0.001$ \\
\hline $\begin{array}{l}\text { High-density lipoprotein cholesterol (HDL-C) } \\
\text { [mmol/L] }\end{array}$ & $1.55 \pm 0.32$ & $1.72 \pm 0.34$ & $1.60 \pm 0.31$ & $1.47 \pm 0.29$ & $1.39 \pm 0.23$ & $<0.001$ \\
\hline Non-HDL & $3.92 \pm 0.99$ & $3.29 \pm 0.80$ & $3.84 \pm 0.87$ & $4.13 \pm 0.91$ & $4.48 \pm 1.01$ & $<0.001$ \\
\hline $\mathrm{TC} / \mathrm{HDL}$ & $3.62 \pm 0.79$ & $2.97 \pm 0.55$ & $3.47 \pm 0.66$ & $3.87 \pm 0.69$ & $4.26 \pm 0.63$ & $<0.001$ \\
\hline TG / HDL & $0.79(0.53-1.30)$ & $0.42(0.34-0.50)$ & $0.67(0.58-0.78)$ & $1.02(0.86-1.20)$ & $1.81(1.5 I-2.28)$ & $<0.001$ \\
\hline $\mathrm{nHDL} / \mathrm{HDL}$ & $2.62 \pm 0.79$ & $1.97 \pm 0.55$ & $2.47 \pm 0.66$ & $2.87 \pm 0.69$ & $3.26 \pm 0.63$ & $<0.001$ \\
\hline
\end{tabular}

Abbreviation: HOMA-IR, homeostatic model assessment of insulin resistance. 
parameters (TC, $\log$ TG, HDL, nHDL, TC/HDL, logTG/ HDL and nHDL/HDL) and CKD. Table 2 shows the relationship between the quartile of the meaningful lipid parameters and the CKD endpoint. In Model 1, age, gender, smoking, drinking, education, exercise, hypertension, diabetes, obesity and hyperuricemia, as confounding factors, were adjusted. The results showed that the fourth quartile of $\log$ TG/HDL was significantly more associated with CKD compared with the first quartile $(\mathrm{OR}=2.453$, $\mathrm{CI}=1.468-4.099, \mathrm{p}<0.001)$. Model 2 was adjusted for confounding factors and hsCRP, and the results showed consistency with Model 1. In Model 3, the confounding factors and HOMA-IR were adjusted at the same time, while the risk of CKD in the fourth quartile of $\log \mathrm{TG} / \mathrm{HDL}$ was weakened $(\mathrm{OR}=2.034, \mathrm{CI}=1.189-3.481, \mathrm{p}<0.05)$. In Model 4, the confounding factors hsCRP and HOMAIR were simultaneously adjusted, and the results were the same as those in Model 3.

During the 5-year follow-up, 163 (15.7\%) participants progressed to CKD. Figure 1 shows the relationship between the CKD incidence and the quartile of baseline $\log$ TG/HDL. Compared with the lower quartile, the highest quartile of $\log$ TG/HDL had a higher incidence rate of CKD $(\mathrm{p}<0.05)$.

Subgroup analysis was performed to examine the relationship between logTG/HDL and CKD according to the factors of gender, obesity, hypertension, hyperuricemia, and dysregulated glucose metabolism. In addition to the non-obese, non-hyperuricemia and male groups, there was a significant difference in the association between $\log \mathrm{TG} / \mathrm{HDL}$ and CKD in the other groups $(\mathrm{P}<0.05)$. In the glucose metabolism disorder group, $\log \mathrm{TG} / \mathrm{HDL}$ was used to predict the occurrence of
CKD with an OR value of 11.45 , while in the non-glycogen metabolism disorder group, the OR value was 2.31. Similarly, in the women, obesity, hypertension, and hyperuricemia groups, the OR values to predict CKD increased were 4.18, $6.26,2.89$ and 8.54 respectively (Figure 2).

The multivariate linear regression method was used to further analyze the relationship of lipid parameters and eGFR and $\log$ ACR in the above-mentioned study population without CKD in a longitudinal analysis. The correlation of eGFR/logACR with statistically significant lipid parameters is shown in Table 3. In the adjusted model, $\log \mathrm{TG}$ (coefficient $=$ $-7.784,95 \%$ CI: $-14.176-1.393, \mathrm{P}<0.05)$ and nHDL/HDL-C (coefficient $=-3.448,95 \% \mathrm{CI}:-5.289-1.608, \mathrm{P}<0.001$ ) were negatively correlated with the participants' eGFR. Log TG (coefficient $=0.136,95 \%$ CI: $0.038-0.234, \mathrm{P}<0.05)$ and TC (coefficient $=0.022,95 \%$ CI: $0.0002,0.043, \mathrm{P}<0.05$ ) were positively correlated with $\log \mathrm{ACR}$.

The ROC curve in Figure 3 shows the ability of three baseline lipid parameters (TC, $\lg \mathrm{TG}$, and $\lg \mathrm{TG} / \mathrm{HDL}$ ) to predict the occurrence of CKD in the study population. Among them, the area under the curve of $\lg \mathrm{TG} / \mathrm{HDL}$ was 0.623 ( $\mathrm{p}<$ 0.001; 95\% CI: 0.593-0.653; cutoff value: 0.236; sensitivity: 46.01\%; specificity: 77.57\%). The areas under the curve of TC and $\operatorname{lgTG}$ are $0.530(\mathrm{p}=0.208$; 95\% CI: $0.483-0.577$; cut-off value: 0.080 ; sensitivity: $57.67 \%$; specificity: $50.34 \%$ ) and 0.611 ( $\mathrm{p}<0.001,95 \%$ CI: 0.580 -0.641; cut-off value: 0.211 ; sensitivity: 42.94\%; specificity: $78.15 \%$ ).

\section{Discussion}

This study was different from previous studies, as it was based on healthy people without CKD to evaluate the ability of lipid parameters to predict $\mathrm{CKD}$, as well as to prevent confusion

Table 2 The Odds Ratio of Chronic Kidney Disease Predicted by Lipid Parameters

\begin{tabular}{|c|c|c|c|c|}
\hline \multirow{2}{*}{$\begin{array}{l}\text { logTG/HDL-C Ratio } \\
\text { vs I st quartile }\end{array}$} & \multicolumn{4}{|c|}{ Odds Ratios (95\% Confidence Intervals) } \\
\hline & $\begin{array}{l}\text { Model I } \\
\text { Adjusted for } \\
\text { Demographics }\end{array}$ & $\begin{array}{l}\text { Model } 2 \\
\text { Adjusted for } \\
\text { Demographics + } \\
\text { HsCRP }\end{array}$ & $\begin{array}{l}\text { Model } 3 \\
\text { Adjusted for } \\
\text { Demographics + } \\
\text { HOMA-IR }\end{array}$ & $\begin{array}{l}\text { Model } 4 \\
\text { Adjusted for Demographics + } \\
\text { hsCRP + HOMA-IR }\end{array}$ \\
\hline$P$ for trend & $P<0.001$ & $\mathrm{P}<0.00 \mathrm{I}$ & $P<0.00$ I & $P<0.001$ \\
\hline 2nd quartile & $0.967(0.543-1.722)$ & $0.967(0.543-1.722)$ & $0.93 I(0.523-1.660)$ & $0.93 I(0.523-1.660)$ \\
\hline 3rd quartile & $1.178(0.675-2.056)$ & $1.178(0.675-2.056)$ & $1.061(0.602-1.869)$ & $1.061(0.602-1.869)$ \\
\hline 4th quartile & $2.453 * *(1.468-4.099)$ & $2.453 * *(1.468-4.099)$ & $2.034 *(1.189-3.48 I)$ & $2.034 *(1.189-3.48 I)$ \\
\hline
\end{tabular}

Note: $* \mathrm{P}<0.05 ; * * \mathrm{P}<0.001$.

Abbreviations: TG/HDL, ratio of triglycerides to non-high-density lipoprotein; HsCRP, hypersensitive C-reactive protein; HOMA-IR, homeostatic model assessment of insulin resistance. 


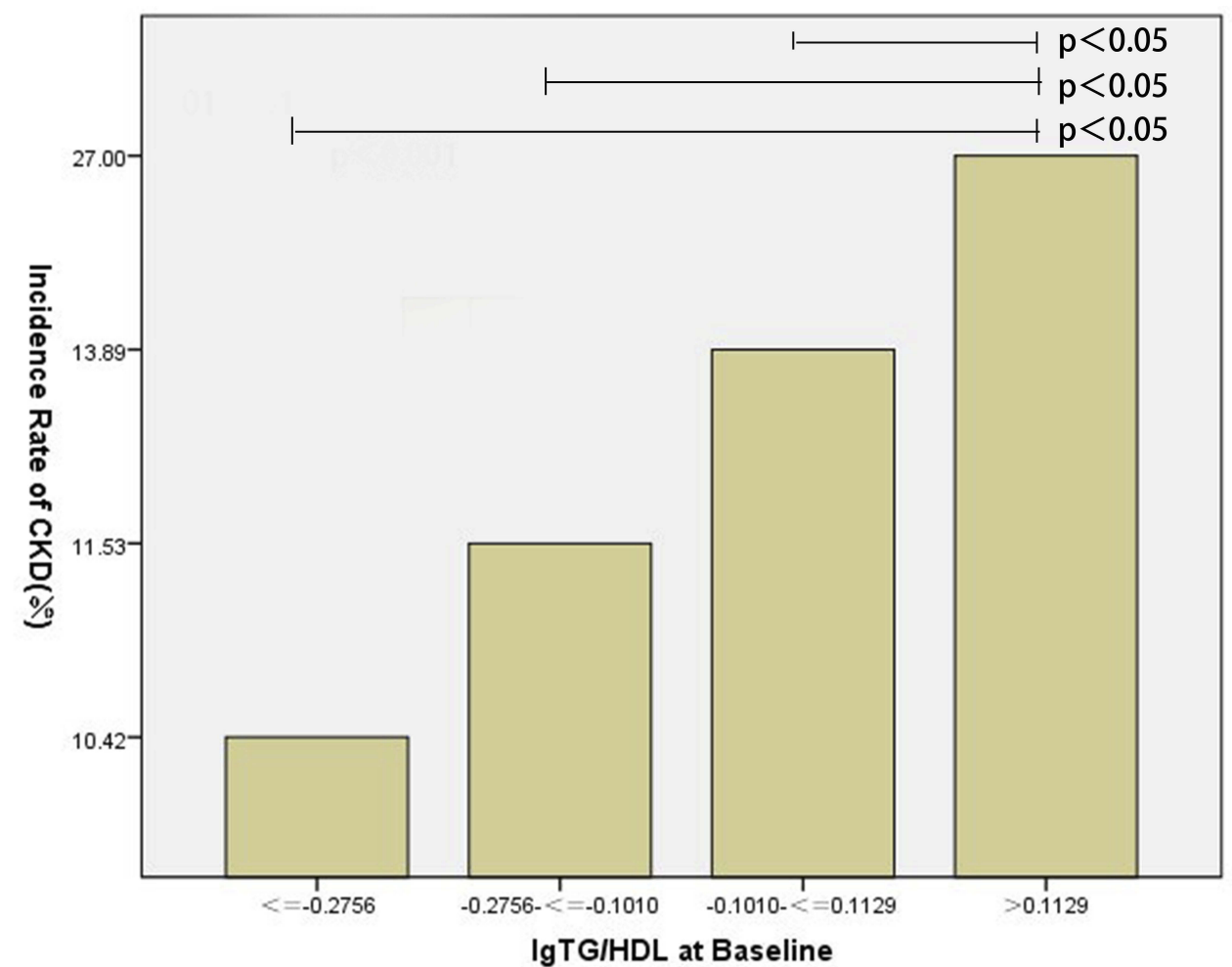

Figure I The incidence rate of CKD stratified by quartiles of logTG/HDL at baseline.

Abbreviations: CKD, chronic kidney disease; TG/HDL, ratio of triglycerides to non-high-density lipoprotein.

\section{Odds Ratio per Standard Deviation Higher IgTG/HDL}

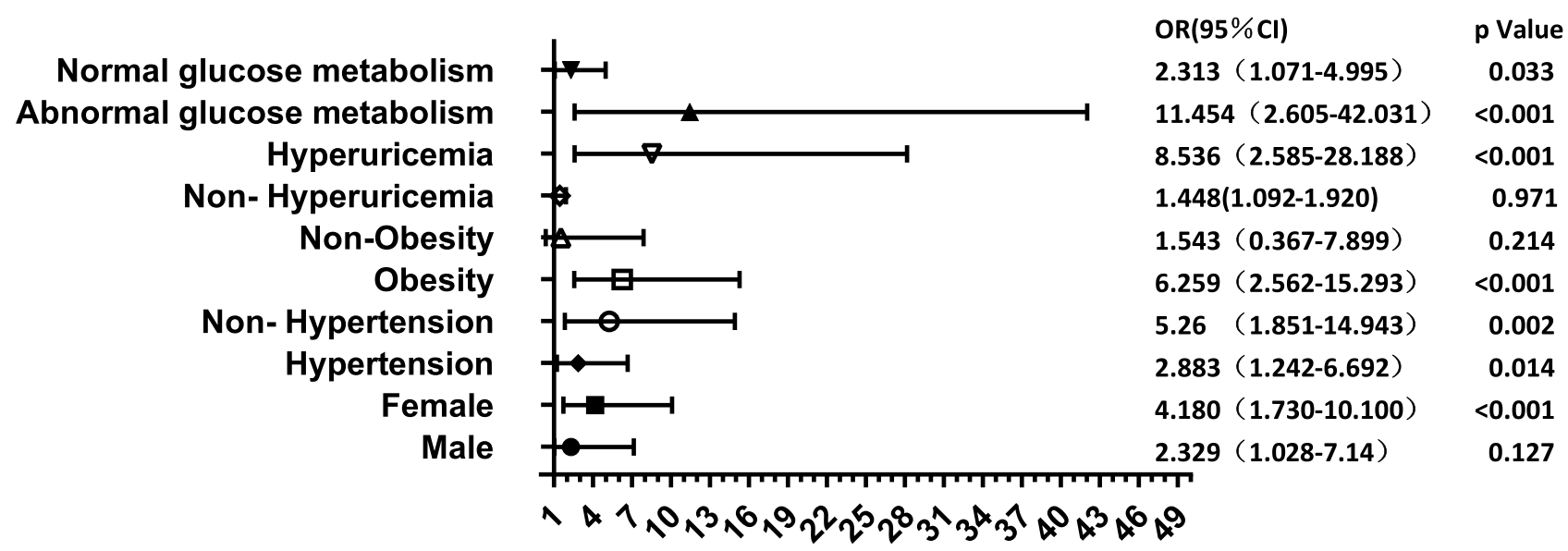

Figure 2 The odds ratio of CKD according to logTG/HDL in the gender, hypertension, obesity, hyperuricemia and glucose metabolism disorder subgroups. The model was adjusted for the above confounding factors, except for grouping variables. The error bars delineate the $95 \%$ confidence interval.

Abbreviation: TG/HDL, ratio of triglycerides to non-high-density lipoproteins.

regarding the effect of CKD on lipid metabolism disorders. In addition, to our knowledge, this study was the first to report a positive correlation between baseline nHDL/HDL and $\log \mathrm{ACR}$ in healthy populations in longitudinal studies.

In this longitudinal study based on the Chinese population, our findings were as follows: (I) $\log \mathrm{TG} / \mathrm{HDL}$ was associated with an increased risk of CKD. (II) We also evaluated the inflammation measured by hsCRP and the insulin resistance measured by HOMA-IR to explain the underlying mechanism of the association between lipid parameters and CKD. (III) Among the lipid parameters we studied, log TG and TC were negatively correlated 
Table 3 Correlation of Estimated Glomerular Filtration Rate (eGFR) and Logarithm of Urinary Albumin to Creatinine Ratio ( $\log A C R)$ with Lipid Parameters

\begin{tabular}{|l|l|l|l|l|}
\hline \multirow{2}{*}{ Variables } & \multicolumn{2}{|l|}{ lGFR } & \multicolumn{2}{l|}{ logACR } \\
\cline { 2 - 5 } & Coefficient $(\mathbf{9 5} \% \mathbf{~ C l})$ & P value & Coefficient $\mathbf{( 9 5 \% ~ C l )}$ & P value \\
\hline logTG & $-7.784(-14.176-1.393)$ & 0.017 & $0.136(0.038-0.234)$ & 0.007 \\
TC & - & - & $0.022(0.0002-0.043)$ & 0.047 \\
nHDL/HDL ratio & $-3.448(-5.289-1.608)$ & P<0.001 & - & - \\
\hline
\end{tabular}

Abbreviations: TG, triglycerides; TC, total cholesterol; nHDL/HDL, non-high-density lipoprotein to high-density lipoprotein ratio; eGFR, estimated glomerular filtration rate; $A C R$, microalbumin to creatinine ratio.

with eGFR, while logTG and nHDL/HDL were positively correlated with IogACR. (IV) In the analysis of various factors, namely, glucose metabolism disorder, hypertension, hyperuricemia and obesity, $\log$ TG/HDL and the risk of CKD were inconsistent, which suggested that these factors may synergistically affect the association between lipids and CKD. (V) ROC curve analysis showed that lgTG/HDL has a higher predictive value for CKD than other lipid indicators (lgTG and TC).

Abnormalities in lipid parameter levels could lead to impaired renal function. Our findings were consistent with previous reports. Ho et $\mathrm{al}^{5}$ showed that the TG/HDL-C ratio was associated with adverse CKD risk profiles in healthy populations, but the study lacked a longitudinal comparison of the predicted value of TG/HDL-C on CKD progression over time. A cross-sectional study based on a large population in Japan showed that higher TG/HDL-C levels were associated with an increased risk of $\mathrm{CKD}$, especially in participants with hypertension, diabetes and obesity. ${ }^{6}$ The TG/HDL-C ratio was a better marker of renal impairment compared with other conventional lipids, ${ }^{7}$ and our study also found that only $\log \mathrm{TG} / \mathrm{HDL}$ is independently associated with CKD. In the disorder of lipid metabolism, elevated TG, TC and nonHDL levels accelerated atherosclerosis, while HDL, with the functions of anti-oxidative stress, inhibition of inflammation and anti-thrombosis,

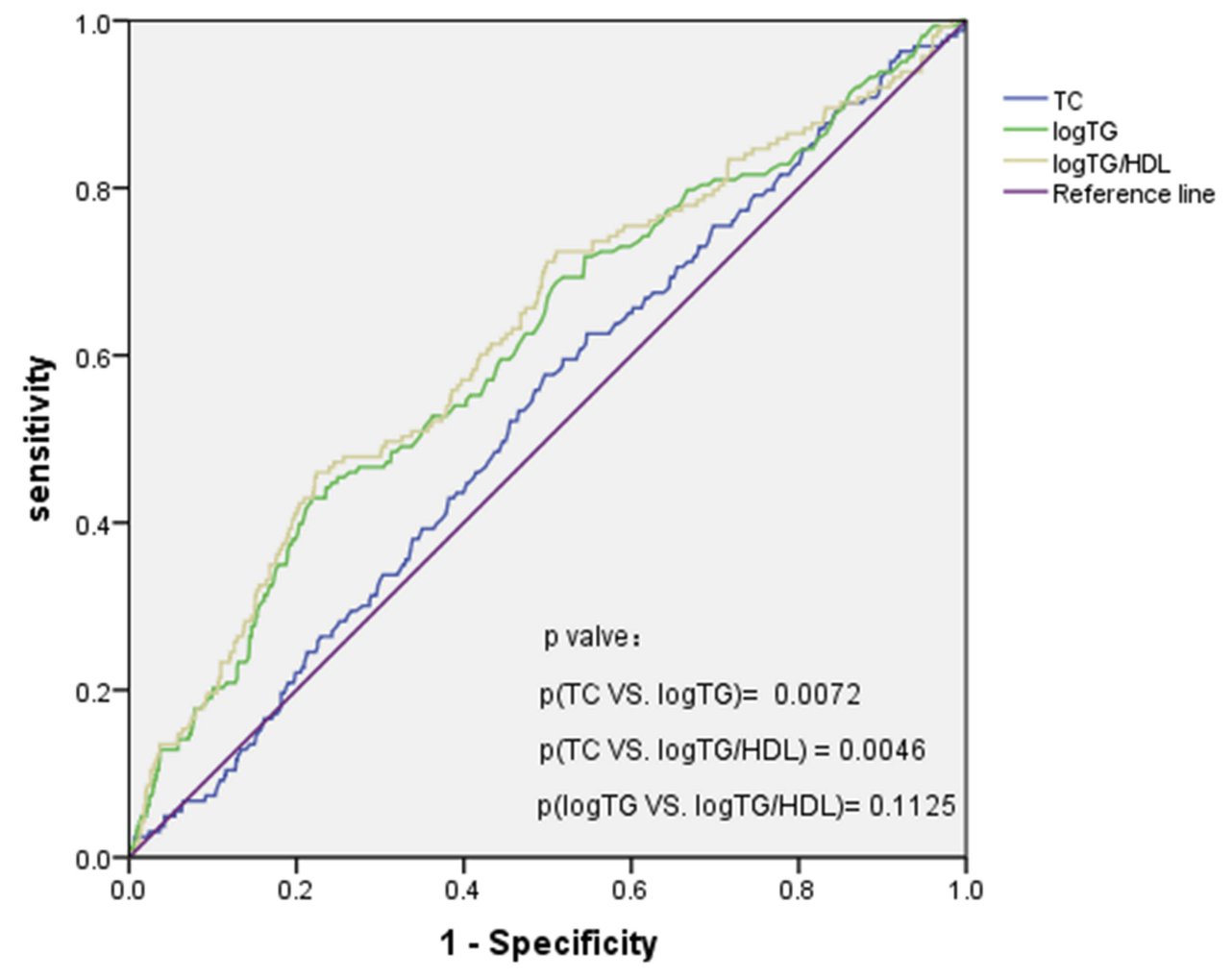

Figure 3 ROC curve analysis of TC, $\lg T G$ and $\lg T G / H D L$ values to predict CKD.

Abbreviations: TC, total cholesterol; TG, triglycerides; TG/HDL, ratio of triglycerides to non-high-density lipoprotein. 
tended to decrease atherosclerosis, which together promoted the development of CKD. ${ }^{8}$ TC and TG levels were negatively correlated with eGFR at different ages. ${ }^{9,10}$ In a prospective observational study in a population with CKD, LDL-C in patients with moderate to low proteinuria was closely associated with total cholesterol levels and poor prognosis of kidney disease. ${ }^{11}$ As a novel indicator, a higher NonHDLc/HDLc ratio predicted the risk of new-onset CKD and had a slight significant correlation with low eGFR, which could serve as a potential indicator for identifying high-risk populations with $\mathrm{CKD}$; however, the relationship between the NonHDLc/HDLc ratio and ACR was not reported. ${ }^{12}$ In a large cohort study of type 2 diabetes, higher plasma HDL-C levels were associated with a lower risk of CKD events during a 5-year median follow-up. All of these studies have shown that dyslipidemia can impair kidney function, leading to a decrease in eGFR and an increase in proteinuria, which ultimately leads to CKD events. ${ }^{13}$

As the baseline logTG/HDL increased, individuals had a higher risk of CKD, and when HOMA-IR was included in the model, $\log$ TG/HDL was less likely to predict CKD risk (Models 3 and 4 in Table 2), although hsCRP was not. This suggested a possible link between $\log$ TG/HDL and CKD through HOMA-IR. The accumulation of kidney fat could be used to explain the impairment of kidney function caused by lipid disorders. Systemic insulin resistance and hyperlipidemia could promote renal lipid uptake or local fatty acid synthesis, causing structural and functional changes in mesangial cells, podocytes and proximal tubule cells, leading to apoptosis and differentiation, which contributes to the development of fibrosis and CKD. ${ }^{14,15}$ Meanwhile, inflammation, reactive oxygen species (ROS), endogenous electrical stress and other pathophysiological mechanisms caused by hyperlipidemia would also promote the pathological changes of mitochondria and trigger the apoptotic pathway. ${ }^{14}$ In the process of lipid dysfunction leading to $\mathrm{CKD}$, the destruction of cholesterol homeostasis in podocytes played an important role. The accumulation of cellular cholesterol due to increased synthesis, inflow or reduced efflux led to the destruction of the filtered glomerular barrier and the development of proteinuria nephropathy. ${ }^{16}$ At the same time, the decline of HDL with the reverse transport of cholesterol, anti-oxidation, anti-inflammatory and anti-apoptotic effects inhibited the development of CKD. ${ }^{8,17}$

As one of the components of metabolic syndrome, hyperlipidemia is often accompanied by hypertension, impaired glucose tolerance, obesity and other clinical manifestations.
Therefore, in this study, $\log$ TG/HDL was also investigated in the subgroup of hypertension and glucose metabolism disorders, namely, obesity and hyperuricemia, regarding the risk of CKD. Our results suggested that the risk of CKD is enhanced with an increase in MetS-related factors, although no similar conclusions were reached in the hypertension group. Tanaka $\mathrm{H}$ et al reported a positive correlation between the number of components of metabolic syndrome and the prevalence of CKD. ${ }^{18}$ Ninomiya $\mathrm{T}$ et al also confirmed this association. ${ }^{19}$ KitiyakaraÇ et al, in a Southeast Asian cohort, showed that the metabolic syndrome defined by NCEP ATP III was associated with a new risk of CKD. ${ }^{20}$ In addition, studies have shown that elevated serum uric acid levels were an independent risk factor for kidney disease. ${ }^{21,22}$ Other components of Mets cause microalbuminuria, angiogenesis, mesangial cell proliferation and extracellular matrix expansion through increased glomerular filtration, renal endothelial dysfunction, activation of the renin-angiotensin system (RAS), oxidative stress and abnormal secretion of growth factors. ${ }^{23}$

This study has some limitations. First, because we only studied residents who were willing to participate, those with more comorbidities and those who did not have jobs may have had a higher participation rate. In addition, compared with Western countries, the population we studied was younger, which may lead to selection bias. Second, the lack of simultaneous assessment of apolipoprotein A and apolipoprotein $\mathrm{B}$ and other lipid parameters and the ability to predict CKD is another limitation of this study. Third, creatinine is a product of muscle metabolism. The creatininebased eGFR we assessed to evaluate renal function may not be accurate for assessing middle-aged and elderly people. Fourth, the development of CKD within two years of initial testing seems to limit the sensitivity of the study. Finally, the relatively short mean follow-up time may not have been sufficient to show the impact of dyslipidemia on renal function in a relatively young cohort, and a longer follow-up in older people may have shown a greater number of patients with renal involvement.

\section{Conclusions}

In conclusion, we report that only baseline $\log \mathrm{TG} / \mathrm{HDL}$ is an independent risk factor for CKD, and $\log \mathrm{TG} / \mathrm{HDL}$ may be useful for clinical monitoring of CKD. In the obese and female populations, as well as in those with disorders of glucose metabolism and hyperuricemia, this risk is exacerbated, so special attention needs to be paid to lipid control in these populations. We report that log TG and nHDL/ HDL-C are negatively correlated with eGFR. Log TG and 
TC are positively correlated with $\log$ ACR. Further research is needed to replicate these findings in other cohorts and to assess the pathophysiological basis and significance.

\section{Funding}

This study was supported by the "Risk factors and prediction model of chronic kidney disease caused by metabolic syndrome: A multicentric prospective cohort study" clinical trial training project of Southern Medical University (LC2016PY047, 2016), Science and Technique Program of Guangzhou (201604020015, 2015), South Wisdom Valley Innovative Research Team Program (CXTD-004, 2014), and The National Natural Science Foundation of China (81873620).

\section{Disclosure}

The authors report no conflicts of interest in this work.

\section{References}

1. Go AS, Chertow GM, Fan D, McCulloch CE, Hsu CY. Chronic kidney disease and the risks of death, cardiovascular events, and hospitalization. N Engl J Med. 2004;351(13):1296-1305.

2. McCullough KP, Morgenstern H, Saran R, Herman WH, Robinson BM. Projecting ESRD incidence and prevalence in the United States through 2030. J Am Soc Nephrol. 2019;30(1):127-135. doi:10.1681/ASN. 2018050531

3. Libby P, Ridker PM, Maseri A. Inflammation and atherosclerosis. Circulation. 2002;105(9):1135-1143. doi:10.1161/hc0902.104353

4. Singh R, Kaushik S, Wang Y, et al. Autophagy regulates lipid metabolism. Nature. 2009;458(7242):1131-1135. doi:10.1038/nature07976

5. Ho CI, Chen JY, Chen SY, et al. Relationship between TG/ HDL-C ratio and metabolic syndrome risk factors with chronic kidney disease in healthy adult population. Clin Nutr. 2015;34(5):874-880. doi:10.1016/j.clnu.2014.09.007

6. Tsuruya K, Yoshida H, Nagata M, et al. Association of the triglycerides to high-density lipoprotein cholesterol ratio with the risk of chronic kidney disease: analysis in a large Japanese population. Atherosclerosis. 2014;233 (1):260-267. doi:10.1016/j.atherosclerosis.2013.12.037

7. Sun K, Lin D, Li F, et al. Discordant associations of lipid parameters with albuminuria and chronic kidney disease: a population-based study. Lipids Health Dis. 2015;14:152. doi:10.1186/s12944-015-0153-8
8. Vaziri ND, Norris K. Lipid disorders and their relevance to outcomes in chronic kidney disease. Blood Purif. 2011;31(1-3):189-196. doi: $10.1159 / 000321845$

9. Hou X, Wang C, Zhang X, et al. Triglyceride levels are closely associated with mild declines in estimated glomerular filtration rates in middle-aged and elderly Chinese with normal serum lipid levels. PLoS One. 2014;9(9):e106778. doi:10.1371/journal.pone.0106778

10. Moafi M, Assadi F, Heshmat R, et al. Impact of dyslipidemia on estimated glomerular filtration rate in apparently healthy children and adolescents: the CASPIAN-V study. World J Pediatr. 2019. doi:10.1007/s12519-019-00270-2

11. Rahman M, Yang W, Akkina S, et al. Relation of serum lipids and lipoproteins with progression of CKD: the CRIC study. Clin J Am Soc Nephrol. 2014;9(7):1190-1198. doi:10.2215/CJN.09320913

12. Zuo PY, Chen XL, Liu YW, Zhang R, He XX, Liu CY. Non-HDLcholesterol to HDL-cholesterol ratio as an independent risk factor for the development of chronic kidney disease. Nutr Metab Cardiovasc Dis. 2015;25(6):582-587. doi:10.1016/j.numecd.2015.03.003

13. Zoppini G, Targher G, Chonchol M, Perrone F, Lippi G, Muggeo M. Higher HDL cholesterol levels are associated with a lower incidence of chronic kidney disease in patients with type 2 diabetes. Nutr Metab Cardiovasc Dis. 2009;19(8):580-586. doi:10.1016/j.numecd.2008.11.003

14. Gai Z, Wang T, Visentin M, Kullak-Ublick GA, Fu X, Wang Z. Lipid accumulation and chronic kidney disease. Nutrients. 2019;11(4). doi: $10.3390 /$ nu 11040722

15. Stadler K, Goldberg IJ, Susztak K. The evolving understanding of the contribution of lipid metabolism to diabetic kidney disease. Curr Diab Rep. 2015;15(7):40. doi:10.1007/s11892-015-0611-8

16. Merscher S, Pedigo CE, Mendez AJ. Metabolism, energetics, and lipid biology in the podocyte - cellular cholesterol-mediated glomerular injury. Front Endocrinol (Lausanne). 2014;5:169.

17. Ferro CJ, Mark PB, Kanbay M, et al. Lipid management in patients with chronic kidney disease. Nat Rev Nephrol. 2018;14(12):727-749.

18. Tanaka H, Shiohira Y, Uezu Y, Higa A, Iseki K. Metabolic syndrome and chronic kidney disease in Okinawa, Japan. Kidney Int. 2006;69 (2):369-374. doi:10.1038/sj.ki.5000050

19. Ninomiya T, Kiyohara Y, Kubo M, et al. Metabolic syndrome and $\mathrm{CKD}$ in a general Japanese population: the Hisayama Study. Am J Kidney Dis. 2006;48(3):383-391. doi:10.1053/j.ajkd.2006.06.003

20. Kitiyakara C, Yamwong S, Cheepudomwit S, et al. The metabolic syndrome and chronic kidney disease in a Southeast Asian cohort. Kidney Int. 2007;71(7):693-700. doi:10.1038/sj.ki.5002128

21. Chonchol M, Shlipak MG, Katz R, et al. Relationship of uric acid with progression of kidney disease. Am J Kidney Dis. 2007;50 (2):239-247. doi:10.1053/j.ajkd.2007.05.013

22. Weiner DE, Tighiouart H, Elsayed EF, Griffith JL, Salem DN, Levey AS. Uric acid and incident kidney disease in the community. $\mathrm{J} \mathrm{Am} \mathrm{Soc}$ Nephrol. 2008;19:1204-1211. doi:10.1681/ASN.2007101075

23. Ruan X, Guan Y. Metabolic syndrome and chronic kidney disease. J Diabetes. 2009;1(4):236-245. doi:10.1111/j.1753-0407.2009.00042.x

Diabetes, Metabolic Syndrome and Obesity: Targets and Therapy

\section{Publish your work in this journal}

Diabetes, Metabolic Syndrome and Obesity: Targets and Therapy is an international, peer-reviewed open-access journal committed to the rapid publication of the latest laboratory and clinical findings in the fields of diabetes, metabolic syndrome and obesity research. Original research, review, case reports, hypothesis formation, expert opinion and commentaries are all considered for publication. The manuscript management system is completely online and includes a very quick and fair peer-review system, which is all easy to use. Visit http://www.dovepress.com/testimonials.php to read real quotes from published authors. 